\title{
Investigation on Formulation Strategies to Mitigate Compression-Induced Destabilization in Supersaturated Celecoxib Amorphous Solid Dispersions
}

Kārlis Bērziņša ${ }^{\mathrm{a}}$, Sara J. Fraser-Miller ${ }^{\mathrm{a}}$, Greg Walker ${ }^{\mathrm{b}}$, Thomas Rades ${ }^{\mathrm{c}}$, Keith C. Gordon ${ }^{{ }^{*}}$

aThe Dodd-Walls Centre for Photonic and Quantum Technologies, Department of Chemistry, University of Otago, Dunedin 9016, New Zealand

${ }^{\mathrm{b} S c h o o l ~ o f ~ P h a r m a c y, ~ U n i v e r s i t y ~ o f ~ O t a g o, ~ D u n e d i n ~ 9016, ~ N e w ~ Z e a l a n d ~}$

'Department of Pharmacy, Faculty of Health and Medical Sciences, University of Copenhagen, Copenhagen 1165, Denmark

*Corresponding author

\section{Supporting information}

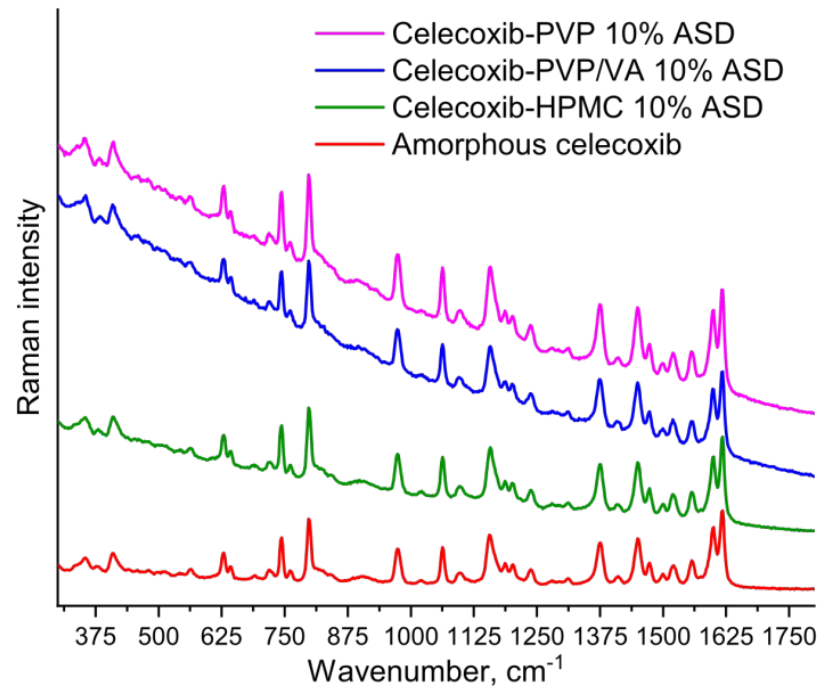

Figure S1. Representative mid-frequency Raman spectra of melt-quenched amorphous celecoxib as well as celecoxib-HPMC, celecoxib-PVP/VA and celecoxib-PVP ASDs with 10\% w/w polymer loading.

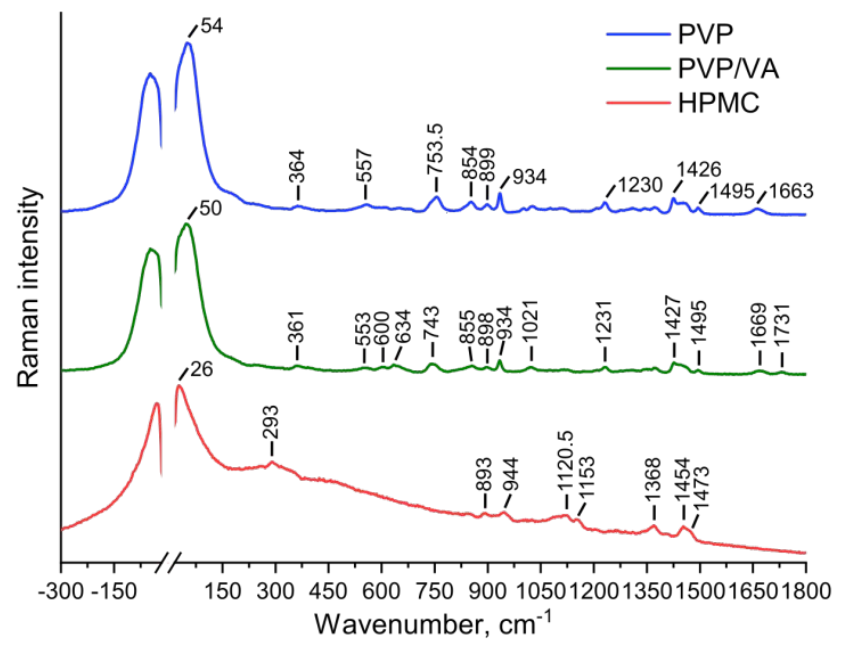


Figure S2. Representative Raman spectra of the utilized HPMC, PVP/VA and PVP. The laserline (Rayleigh scattering) is omitted for clarity.

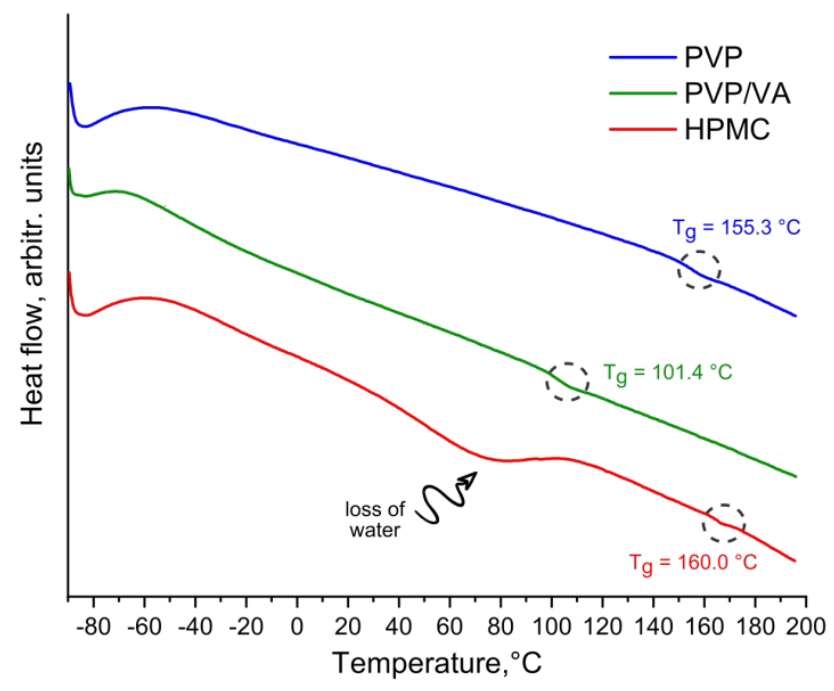

Figure S3. Representative DSC curves of HPMC, PVP/VA and PVP. 

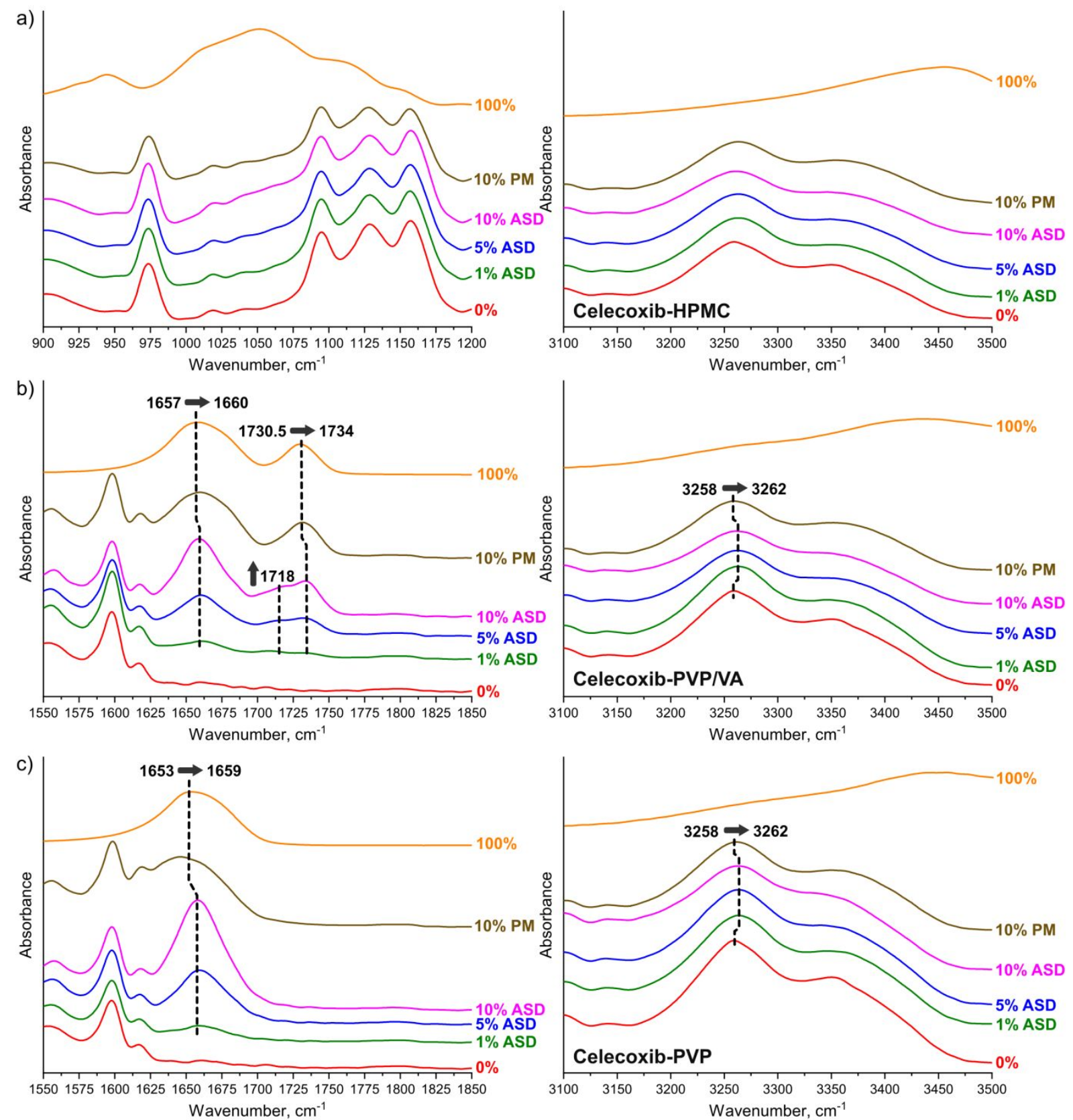

Figure S4. IR spectra of (a) celecoxib-HPMC, (b) celecoxib-PVP/VA and (c) celecoxib-PVP systems, including their physical mixtures (PMs) and individual components in the amorphous state. The polymer concentrations are expressed as $\% \mathrm{w} / \mathrm{w}$. 

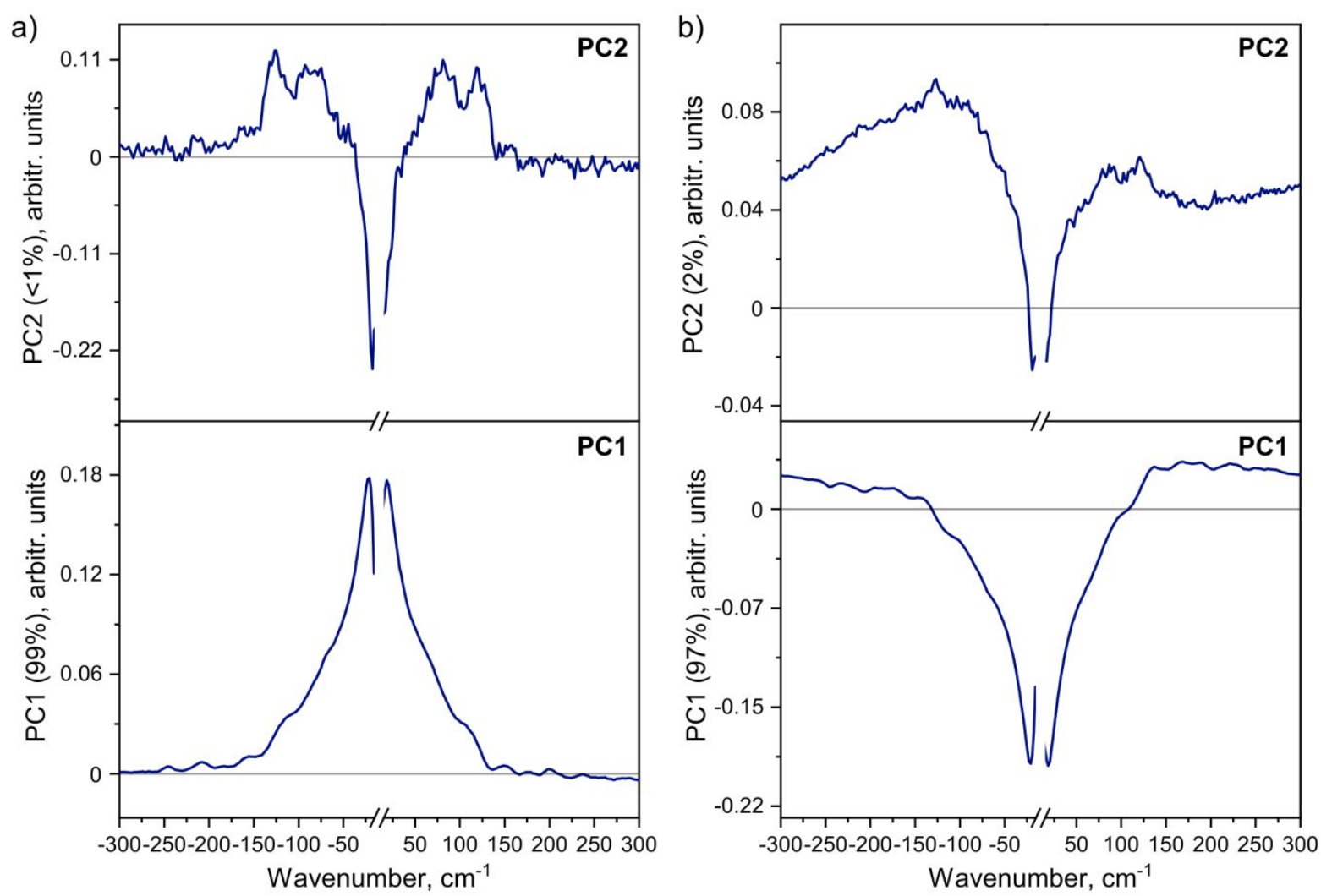

Figure S5. Examples of PCA loadings plots for VTLFR data collected in the range of $-150{ }^{\circ} \mathrm{C}$ to $70{ }^{\circ} \mathrm{C}$ for (a) amorphous celecoxib and (b) celecoxib-PVP 10\% ASD samples.

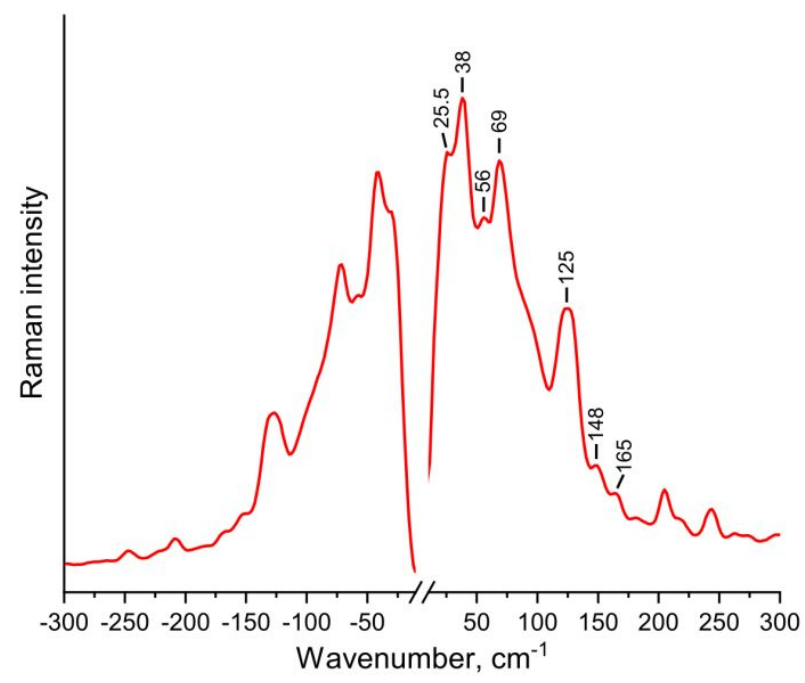

Figure S6. Representative LFR spectrum (room temperature, $\sim 20^{\circ} \mathrm{C}$ ) of celecoxib form IV embedded in an amorphous matrix after partial crystallization from celecoxib-HPMC 5\% ASD sample. 


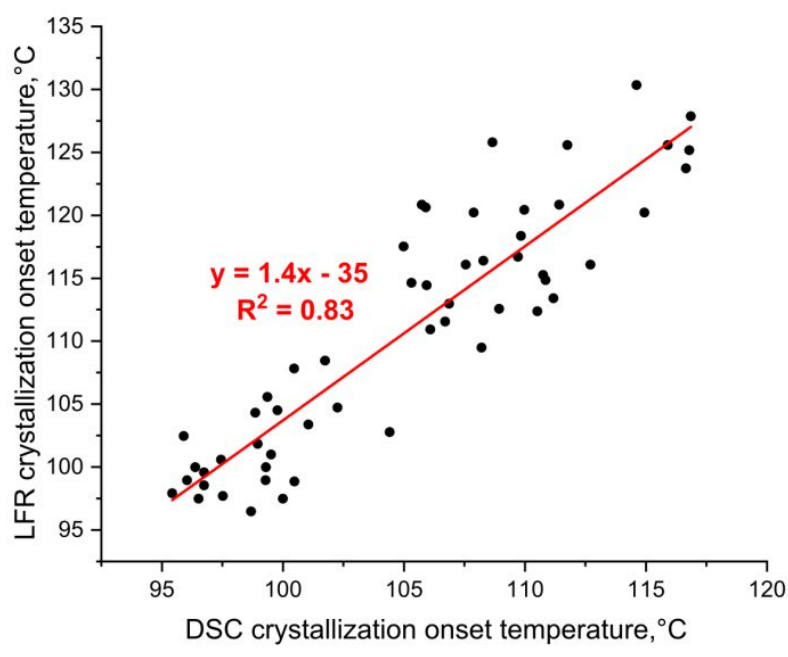

Figure S7. Comparison of the determined crystallization onset temperatures (where applicable) from DSC and LFR measurements for amorphous celecoxib and celecoxib ASD samples. 